'Departamento de Ciencias Básicas. Universidad Católica de la Santísima Concepción. Concepción, Chile. ${ }^{2}$ BHF Glasgow Cardiovascular Research Centre, Institute of Cardiovascular and Medical Sciences, University of Glasgow. Glasgow, United Kingdom. 'Departamento de Ciencias del Deporte y Acondicionamiento Físico, Universidad Católica de la Santísima Concepción. Concepción, Chile.

${ }^{4}$ Escuela de Educación Física, Universidad San Sebastián. Concepción, Chile. ${ }^{5}$ Departamento de Nutrición y Dietética, Facultad de Farmacia, Universidad de Concepción. Concepción, Chile. ${ }^{6}$ Departamento de Bioquímica Clínica e Inmunología, Facultad de Farmacia y Centro de Vida Saludable de la Universidad de Concepción. Concepción, Chile. 'Departamento de Nutrición, Diabetes y Metabolismo, Escuela de Medicina, Pontificia Universidad Católica de Chile. Santiago, Chile.

8Instituto de Nutrición y Tecnología de los Alimentos, Universidad de Chile. Santiago,

Chile

${ }^{9}$ Centro de Investigación en Fisiología de Ejercicio-Cl FE, Universidad Mayor. Santiago, Chile. aBioquímico. ${ }^{\mathrm{b}}$ Nutricionista. cProfesor de Educación Física. dLicenciado en Ciencias Biológicas. ePhD. ${ }^{\mathrm{f}} \mathrm{MSC}$.

* MV y FP contribuyeron de igual manera en este manuscrito y deben considerarse primer autor compartido.

Trabajo no recibió financiamiento. Los autores declaran no tener conflictos de interés.

Recibido el 16 de abril de 2018, aceptado el 15 de noviembre de 2018 .

Correspondencia a: Dr. Carlos Celis-Morales BHF Glasgow Cardiovascular Research Centre. 126 University Avenue, Glasgow. University, Glasgow. United Kingdom. G12 8TA. carlos.celis@glasgow.ac.uk

\section{Asociación entre el polimorfismo rs9939609 del gen FTO con la ingesta energética, macronutrientes y consumo de alcohol en población chilena}

\author{
MARCELO VILLAGRÁN ${ }^{1, a, e, *}$, FANNY PETERMANN-ROCHA ${ }^{2, \mathrm{~b}, \mathrm{f}^{*}}$, \\ LORENA MARDONES ${ }^{1, \mathrm{a}, \mathrm{e}}$, ALEX GARRIDO-MÉNDEZ $^{3,4, \mathrm{c}, \mathrm{e}}$, \\ MIQUEL MARTORELL ${ }^{5, \mathrm{a}, \mathrm{e}}$, NATALIA ULLOA ${ }^{6, \mathrm{a}, \mathrm{e}}$, \\ JOSÉ LUIS SANTOS ${ }^{7, \mathrm{~d}, \mathrm{e}}$, FRANCISCO PÉREZ-BRAVO ${ }^{8, \mathrm{a}, \mathrm{e}}$, \\ CARLOS CELIS-MORALES ${ }^{2,9, c, e, ~}$, en representación \\ del grupo de investigación ELHOC \\ (Epidemiology of Lifestyle and Health Outcomes in Chile)
}

\section{Association of the FTO (rs9939609) genotype with energy intake}

Background: Fat-mass-associated-gene (FTO) is associated with higher energy intake and specific food preferences. Aim: To investigate the association of the FTO genotype with energy intake, macronutrient and alcohol consumption. Material and Methods: Four hundred and nine participants of the GENADIO (Genes, Environment, Diabetes and Obesity) study were included. Energy intake, macronutrient and alcohol consumption were the outcomes of interest. The association of FTO (rs9939609) genotype with these outcomes was investigated using linear regression analyses, adjusting for confounding variables. Results: After adjusting for socio-demographic factors, being a carrier of the risk allele for the FTO gene was associated with a higher energy intake (173 kcal per each extra copy of the risk variant [95\% confidence intervals (CI): 45; 301], $(P=0.008)$. After adjusting for lifestyle factors and body mass index, the association was slightly attenuated but remained significant (144 kcal [95\% CI: 14; 274], $p=0.030$ ). Conclusions: The FTO genotype is associated with a higher energy intake.

(Rev Med Chile 2018; 146: 1252-1260)

Key words: Alpha-Ketoglutarate-Dependent Dioxygenase; Diet; Energy Intake; FTO; Genotype.
L a prevalencia de la obesidad se ha cuadruplicado en las 4 últimas décadas ${ }^{1}$, estimándose que, en el año 2016, más de 650 millones de adultos eran obesos y que 2,8 millones de las muertes anuales fueron causadas por el exceso de peso corporal ${ }^{2}$. El incremento de su prevalencia se ha visto influenciada, fundamentalmente, por los cambios en el estilo de vida, como el aumento de la inactividad física y la adopción de una alimentación no saludable, 
caracterizada por el consumo de alimentos altamente calóricos ${ }^{2-4}$.

Si bien los estilos de vida cumplen un rol fundamental en el desarrollo de la obesidad, estudios realizados en gemelos reportan que la obesidad también posee un componente genético ${ }^{5}$. Es así como durante la última década, numerosos estudios de asociación del genoma (GWAS) han logrado identificar 97 variantes genéticas que predisponen a la obesidad ${ }^{6}$. Entre ellas, el polimorfismo de un nucleótido (SNP) rs9939609 del gen FTO, ha sido uno de los más estudiados debido a que presenta la mayor asociación con marcadores de obesidad, mostrando un aumento de $3 \mathrm{~kg}$ de peso corporal adicionales por cada copia del alelo de riesgo en portadores ${ }^{7}$, lo cual ha sido corroborado en diversas poblaciones, incluyendo chilenos ${ }^{8,9}$. Adicionalmente, diversas líneas de evidencia han vinculado el polimorfismo en FTO con alteraciones en el consumo de alimentos ${ }^{10-15}$. Estudios en ratones han mostrado que FTO se encuentra abundantemente expresado en el núcleo arcuato del hipotálamo, una región reconocida por su rol en la regulación del apetito ${ }^{16}$, y que la reducción de su expresión en el hipotálamo incrementa en 16\% el consumo de alimentos ${ }^{16}$. Por otra parte, estudios epidemiológicos sugieren una asociación positiva entre el genotipo de riesgo de FTO ( $g r F T O$ ) y un alto consumo energético ${ }^{12-15}$, un bajo poder de saciedad ${ }^{10,11}$, una mayor ingesta proteica ${ }^{17}$ y una mayor preferencia por el consumo de grasas ${ }^{12}$. No obstante, hasta ahora la evidencia ha mostrado resultados contradictorios identificando en algunos estudios asociaciones menos robustas o a veces, asociaciones en direcciones opuestas ${ }^{18-20}$.

A pesar de que Chile es uno de los diez países que presenta mayor obesidad a nivel mundial ${ }^{21,22}$, hasta la fecha solo dos estudios han investigado la asociación entre el SNP rs9939609 y el IMC en población infantil y adulta chilena ${ }^{8,9}$. En este contexto, y considerando que el consumo energético es un factor importante vinculado al incremento en los niveles de adiposidad, es relevante investigar si existe alguna asociación entre el grFTO y el consumo de macronutrientes en población Chilena. Esto permitiría corroborar resultados encontrados en otras poblaciones, pero también contribuiría a la generación de nuevas hipótesis de investigación en relación a cuáles podrían ser los mecanismos mediante los cuales el gen FTO se asocia a obesidad. Por lo anterior, el objetivo de este estudio fue investigar la asociación del SNP rs9939609 del gen FTO, con la ingesta energética, de macronutrientes y consumo de alcohol en población adulta chilena.

\section{Materiales y Métodos}

Estudio descriptivo transversal que incluyó a 409 individuos pertenecientes al estudio GENADIO (Genes, Ambiente, Diabetes y Obesidad), realizado en Chile entre los años 2009-2011. La población estudiada estuvo compuesta por 472 residentes de ascendencia mapuche y europea de las regiones del Biobío y Los Ríos (247 mapuche y 225 europea), sin historial médico de enfermedad metabólica o cardiovascular, y que al momento de la evaluación no estuvieran bajo ninguna prescripción de medicamentos, tal como ha sido descrito anteriormente ${ }^{23}$. La muestra fue calculada en base a los datos del CENSO 2002, en el cual la población indígena chilena constituía $4,6 \%$ de la población, siendo los mapuches la etnia más numerosa. El estudio contó con la aprobación de los comités de ética de la Universidad de Chile, Universidad de Concepción y Universidad de Glasgow. Todos los participantes firmaron su consentimiento informado previo a la recolección de datos.

\section{Determinación de variantes alélicas del gen FTO (Fat Mass Obesity-associated gene)}

Para la genotipificación del SNP rs9939609 del gen FTO, se obtuvo ADN genómico de leucocitos periféricos mediante el kit QIAamp DNA blood midi kit (QIAGEN, Ltd. UK). La discriminación alélica se realizó mediante PCR de tiempo real en termociclador ABI 7900-HT usando sondas TaqMan ${ }^{\circledR}$ (Applied Biosystems, Warrington, UK). Todos los análisis de las muestras fueron realizados en duplicados, con $98 \%$ de éxito en la determinación del genotipo.

\section{Variables antropométricas}

El peso y la talla fueron determinados con una balanza electrónica (TANITA TBF 300A, USA) y tallímetro (SECA A800, USA). El perímetro de cintura (PC) fue medido con una cinta métrica no distendible (SECA Modelo 201, USA). El estado nutricional fue clasificado en base a los puntos de corte sugeridos por la OMS ${ }^{24}$. Los valores utilizados para definir obesidad central fueron los 
siguientes: $\mathrm{PC} \geq 102 \mathrm{~cm} \mathrm{y} \geq 88 \mathrm{~cm}$ en hombres y mujeres, respectivamente ${ }^{24}$. La composición corporal se determinó mediante la medición de cuatro pliegues cutáneos (bicipital, sub-escapular, supra-iliaco y tricipital) mediante un caliper Harpenden skinfold (Cranlea \& Company, Birmingham, $\mathrm{UK})^{23}$. Se aplicó la ecuación de Durnin y Womersley para estimar el porcentaje de masa grasa corporal ${ }^{25}$.

\section{Ingesta energética, de macronutrientes y alcohol}

La ingesta dietaria fue evaluada a través del registro y pesado de alimentos y bebidas durante siete días consecutivos. El pesado se realizó en una balanza de cocina digital, para lo cual se instruyó a los participantes con una demostración visual. Se pesaron y descontaron de la ingesta los restos de los alimentos parcialmente consumidos. Se solicitó a los participantes mantener su consumo habitual de alimentos durante este período ${ }^{23}$. La ingesta dietética fue analizada por la Base de Datos de Composición de Alimentos de Chile a través del Software MINUTA de la Universidad de Concepción, mediante el cual se obtuvo el consumo de energía en kcal, de macronutrientes y de alcohol.

\section{Variables sociodemográficas y de estilo de vida}

Los datos sociodemográficos (edad, sexo, zona de residencia, nivel educacional, ingreso económico, etnia) y los datos asociados con estilos de vida, como el tabaquismo, fueron recolectados mediante encuestas validadas ${ }^{23}$.

Para la medición de Fitness cardiorrespiratorio se utilizó el Chester Step Test validado siguiendo las recomendaciones descritas por Buckley et $\mathrm{al}^{26}$. Los niveles de actividad física y el tiempo sedentario fueron estimados por acelerometría de movimiento (Actigraph GTM1, USA). El actígrafo fue adherido a la región de la cadera derecha en cada participante y usado durante 7 días. La intensidad de la actividad física y el gasto energético se determinaron mediante el algoritmo de Freedson ${ }^{27}$.

\section{Análisis estadístico}

Los datos de caracterización de la población estudiada son presentados como promedio y desviación estándar para variables continuas, y como porcentaje para variables categóricas. Las diferencias entre genotipo fueron determinadas con análisis de regresión para variables continuas y con el test Chi-cuadrado para variables categóricas.
Para investigar la asociación entre el polimorfismo rs9939609 del gen FTO, la ingesta energética, de macronutrientes y consumo de alcohol, se realizó análisis de regresión lineal. El genotipo del SNP rs9939609 del gen FTO fue codificado siguiendo un modelo genético aditivo. Esto es, $0=$ TT-homocigoto para el alelo protector; 1 = AT-heterocigoto para el alelo de riesgo; 2 = AA-homocigoto para el alelo de riesgo. Mediante análisis de regresión lineal, se estimó el incremento en macronutrientes y alcohol por cada copia adicional de la variante de riesgo (alelo A). Estos resultados fueron presentados como promedio o coeficiente beta con su respectivo $95 \%$ intervalo de confianza (95\% IC).

Todos los análisis fueron ajustados por variables de confusión mediante la utilización de cuatro modelos estadísticos: Modelo 0 - sin ajustar; Modelo 1 - ajustado por edad, sexo, etnia, nivel educacional, ingreso económico, nivel socioeconómico y zona de residencia (urbano/ rural); Modelo 2 - ajustado por el Modelo 1, pero también por actividad física, tiempo sedente y tabaquismo; Modelo 3 - ajustado por el Modelo 2, pero también por IMC; y Modelo 4 - ajustado por Modelo 2 y PC. Para todos los análisis se utilizó el programa STATA SE v14. El nivel de significancia fue definido como $\mathrm{p}<0,05$.

\section{Resultados}

En la Tabla 1 se presentan las características generales de la población según genotipo (AA, AT, TT). Los individuos con el genotipo AA predominante presentan mayor edad en comparación a individuos asociados a los genotipos AT y TT (6,2 y 8,9 años, respectivamente). En relación con las variables antropométricas, los individuos con genotipo predominante AA poseen un mayor peso corporal, IMC, \% de masa grasa y PC, lo que favorece que exista un mayor porcentaje de individuos obesos y/o con obesidad central en comparación a individuos con genotipo AT y TT. No obstante, no se observaron diferencias significativas en los parámetros de actividad física ni en las variables sociodemográficas entre los individuos con alelos de riesgo respecto a individuos con el genotipo protector (TT).

Los resultados de la asociación entre el SNP rs9939609 del gen FTO, ingesta energética, de macronutrientes y consumo de alcohol son pre- 
Tabla 1. Características de la población según genotipo del gen FTO (rs9939609)

\begin{tabular}{|c|c|c|c|c|}
\hline & TT & AT & AA & Valor $\mathbf{p}$ \\
\hline$n$ & 203 & 167 & 39 & \\
\hline Edad & $35,2 \pm 13,2^{\mathrm{AA}}$ & $37,9 \pm 12,0^{\mathrm{AA}}$ & $44,1 \pm 13,3^{\top T, A T}$ & 0,0003 \\
\hline Sexo, mujeres (\%) & 56,2 & 53,3 & 71,8 & 0,110 \\
\hline Zona geográfica, urbana (\%) & 59,6 & 56,8 & 59,0 & \\
\hline $\begin{array}{l}\text { Etnia (\%) } \\
\text { Europea } \\
\text { Mapuche }\end{array}$ & $\begin{array}{l}43,8 \\
56,2\end{array}$ & $\begin{array}{l}56,9 \\
43,1\end{array}$ & $\begin{array}{l}51,3 \\
48,7\end{array}$ & 0,044 \\
\hline $\begin{array}{l}\text { Nivel Educacional (\%) } \\
\text { Nivel básico } \\
\text { Enseñanza media } \\
\text { Técnico / universitario }\end{array}$ & $\begin{array}{l}23,0 \\
43,7 \\
33,3\end{array}$ & $\begin{array}{l}24,8 \\
42,7 \\
32,5\end{array}$ & $\begin{array}{l}36,4 \\
42,4 \\
21,2\end{array}$ & 0,499 \\
\hline $\begin{array}{l}\text { Ingresos (\%) } \\
\text { Bajo } \\
\text { Medio } \\
\text { Alto }\end{array}$ & $\begin{array}{l}39,8 \\
11,0 \\
49,2\end{array}$ & $\begin{array}{l}38,2 \\
13,4 \\
48,4\end{array}$ & $\begin{array}{c}42,4 \\
9,1 \\
48,5\end{array}$ & 0,943 \\
\hline $\begin{array}{l}\text { Tabaquismo (\%) } \\
\text { No } \\
\text { Sí }\end{array}$ & $\begin{array}{l}50,7 \\
49,3\end{array}$ & $\begin{array}{l}47,9 \\
52,1\end{array}$ & $\begin{array}{l}33,3 \\
66,7\end{array}$ & 0,137 \\
\hline Peso corporal (kg) & $68,6 \pm 9,8^{\mathrm{AT}, \mathrm{AA}}$ & $72,3 \pm 10,4^{T T, A A}$ & $76,5 \pm 9,9^{\Pi, A T}$ & $<0,0001$ \\
\hline IMC $\left(\mathrm{kg} / \mathrm{m}^{2}\right)$ & $27,0 \pm 3,7^{\mathrm{AT}, \mathrm{AA}}$ & $28,5 \pm 3,7^{T T, A A}$ & $30,2 \pm 2,8^{\Pi, A T}$ & $<0,0001$ \\
\hline $\begin{array}{l}\text { Estado Nutricional (\%) } \\
\text { Bajo peso } \\
\text { Normal } \\
\text { Sobrepeso } \\
\text { Obeso }\end{array}$ & $\begin{array}{c}0,4 \\
29,6 \\
49,3 \\
20,7\end{array}$ & $\begin{array}{c}0 \\
17,4 \\
49,7 \\
32,9\end{array}$ & $\begin{array}{c}0 \\
2,6 \\
48,7 \\
48,7\end{array}$ & $<0,0001$ \\
\hline Masa grasa (\%) & $28,8 \pm 4,6^{\mathrm{AA}}$ & $29,9 \pm 4,3^{\mathrm{AA}}$ & $31,6 \pm 3,2^{\pi, A T}$ & 0,0004 \\
\hline Perímetro de cintura $(\mathrm{cm})$ & $95,1 \pm 10,8^{\mathrm{AA}}$ & $97,6 \pm 11,2^{\mathrm{AA}}$ & $101,7 \pm 7,3^{\top T, A T}$ & 0,001 \\
\hline Obesidad central (\%) & 54,7 & 60,5 & 92,3 & $<0,0001$ \\
\hline $\begin{array}{l}\text { Actividad física y fitness } \\
\text { Fitness cardiorrespiratorio (METs) } \\
\text { Actividad física (MET/h/sem) } \\
\text { Tiempo sedentario (min/día) }\end{array}$ & $\begin{array}{c}45,3 \pm 11,5 \\
934,3 \pm 284,8 \\
508,4 \pm 87,6^{\text {AA }}\end{array}$ & $\begin{array}{c}45,5 \pm 12,0 \\
876,3 \pm 281,2 \\
531,8 \pm 92,3\end{array}$ & $\begin{array}{c}42,7 \pm 11,0 \\
891,0 \pm 333,6 \\
549,2 \pm 95,9^{\top \top}\end{array}$ & $\begin{array}{l}0,407 \\
0,272 \\
\mathbf{0 , 0 2 3}\end{array}$ \\
\hline
\end{tabular}

Datos presentados como promedio y desviación estándar para variables continuas y como \% para variables categóricas. Las diferencias entre genotipo fueron determinadas con análisis de regresión para variables continuas y con el test Chi-cuadrado para variables categóricas. Se ha adicionado uno o más de un genotipo AA, AT, TT para diferenciar las variables que son estadísticamente significativas entre sí.

sentados en la Tabla 2. Los resultados revelan que no existió asociación entre la ingesta energética y el gen FTO en el modelo no ajustado. No obstante, al ajustar el modelo por las variables sociodemográficas (Modelo 1), el incremento en el consumo energético fue equivalente a $136 \mathrm{kcal}$ por cada copia extra del alelo de riesgo $(\mathrm{p}=0,015)$. Al ajustar el modelo por actividad física, sedentaris- mo y tabaquismo (Modelo 2), la magnitud de la asociación aumentó, observándose un incremento energético de $173 \mathrm{kcal}$ por cada copia extra del alelo de riesgo $(p=0,008)$. Finalmente, al ajustar el modelo por IMC (Modelo 3) o PC (Modelo 4), la magnitud de la asociación se redujo a 144 kcal y $151 \mathrm{kcal}$ respectivamente, permaneciendo significativa $(\mathrm{p}=0,030$ y $\mathrm{p}=0,020)$ (Figura 1$)$. 


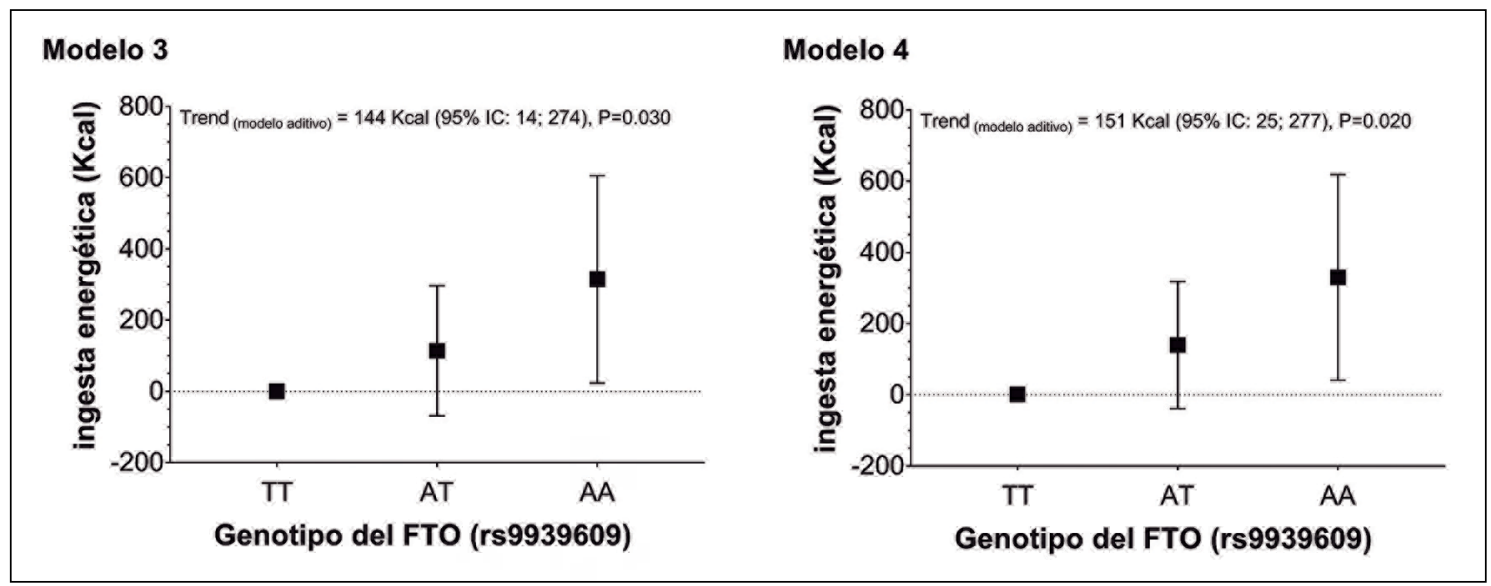

Figura 1. Asociación entre el gen FTO (rs9939609) e ingesta energética. Datos presentados como delta y 95\% IC según genotipo. El modelo genético aditivo indica el promedio de incremento en la variable de adiposidad por cada copia adicional de la variante de riesgo (A). Este efecto aditivo y su respectivo 95\% IC fue determinado mediante regresión lineal. Los análisis fueron ajustados por edad, sexo, etnia, nivel educacional, ingreso económico, nivel socioeconómico, zona de residencia (urbano/rural), actividad física, tiempo sedente, tabaquismo e IMC (Modelo 3) o perímetro de cintura (Modelo 4).

Se identificó una sola asociación entre el polimorfismo y el consumo de grasas poliinsaturadas al ajustar por variables sociodemográficas (Modelo 1) ( $\mathrm{p}=0,039)$; sin embargo, esta asociación des- apareció al realizar un mayor nivel de ajustes por variables de confusión (Tabla 2). Para el resto de los macronutrientes y el consumo de alcohol no se identificó asociación en ninguno de los modelos.

Tabla 2. Asociación entre el gen FTO (rs9939609), ingesta energética, macronutrientes y alcohol

\begin{tabular}{|c|c|c|c|c|c|}
\hline \multirow[b]{2}{*}{ Variables } & \multicolumn{3}{|c|}{ Genotipo del FTO (rs9939609) } & \multirow{2}{*}{$\begin{array}{l}\text { Efecto del modelo } \\
\text { genético aditivo }\end{array}$} & \multirow{2}{*}{$\begin{array}{c}\text { Valor } \\
\text { p }\end{array}$} \\
\hline & TT & AT & AA & & \\
\hline \multicolumn{6}{|c|}{ Ingesta energética (kcal/día) } \\
\hline Modelo 0 & $2.511(2.394 ; 2.628)$ & $2.571(2.442 ; 2.700)$ & $2.722(2.436 ; 3.007)$ & $85(-44 ; 215)$ & 0,196 \\
\hline Modelo 1 & $2.495(2.395 ; 2.595)$ & $2.607(2.498 ; 2.716)$ & $2.797(2.556 ; 3.038)$ & $136(27 ; 246)$ & 0,015 \\
\hline Modelo 2 & $2.481(2.361 ; 2.601)$ & $2.637(2.505 ; 2.769)$ & $2.843(2.584 ; 3.102)$ & $173(45 ; 301)$ & 0,008 \\
\hline Modelo 3 & $2.513(2.396 ; 2.630)$ & $2.606(2.478 ; 2734)$ & $2.778(2.527 ; 3.030)$ & $144(14 ; 274)$ & 0,030 \\
\hline Modelo 4 & $2.493(2.375 ; 2.611)$ & $2.631(2.501 ; 2.760)$ & $2.808(2.553 ; 3.064)$ & $151(25 ; 277)$ & 0,020 \\
\hline \multicolumn{6}{|c|}{ Proteínas (\%ET/día) } \\
\hline Modelo 0 & $14,6(14,0 ; 15,2)$ & $14,3(13,6 ; 14,9)$ & $13,7(12,3 ; 15,2)$ & $-0.35(-1,01 ; 0,31)$ & 0,299 \\
\hline Modelo 1 & $14,7(14,1 ; 15,3)$ & $14,2(13,6 ; 14,9)$ & $14,0(12,5 ; 15,4)$ & $-0,44(-1,10 ; 0,21)$ & 0,184 \\
\hline Modelo 2 & $14,7(14,0 ; 15,4)$ & $14,4(13,7 ; 15,2)$ & $14,2(12,8 ; 15,7)$ & $-0,24(-0,97 ; 0,49)$ & 0,517 \\
\hline Modelo 3 & $14,8(14,1 ; 15,5)$ & $14,4(13,7 ; 15,2)$ & $14,2(12,7 ; 15,6)$ & $-0,32(-1,06 ; 0,42)$ & 0,395 \\
\hline Modelo 4 & $14,7(14,0 ; 15,4)$ & $14,4(13,7 ; 15,2)$ & $14,2(12,7 ; 15,7)$ & $-0,26(-0,99 ; 0,48)$ & 0,492 \\
\hline \multicolumn{6}{|c|}{ Grasas totales (\%ET/día) } \\
\hline Modelo 0 & $25,4(24,1 ; 26,7)$ & $26,1(24,6 ; 27,5)$ & $24,2(21,1 ; 27,3)$ & $-0,07(-1,50 ; 1,35)$ & 0,918 \\
\hline Modelo 1 & $25,3(24,0 ; 26,6)$ & $26,3(24,9 ; 27,8)$ & $24,1(20,9 ; 27,2)$ & $0,05(-1,39 ; 1,49)$ & 0,946 \\
\hline Modelo 2 & $25,3(23,8 ; 26,8)$ & $26,1(24,4 ; 27,7)$ & $24,0(20,8 ; 27,2)$ & $-0,15(-1,75 ; 1,44)$ & 0,850 \\
\hline Modelo 3 & $25,1(23,6 ; 26,6)$ & $26,2(24,5 ; 27,8)$ & $24,2(21,0 ; 27,5)$ & $0,07(-1,55 ; 1,70)$ & 0,930 \\
\hline Modelo 4 & $25,2(23,7 ; 26,7)$ & $26,1(24,4 ; 27,7)$ & $24,2(20,9 ; 27,4)$ & $0,02(-1,60 ; 1,64)$ & 0,982 \\
\hline
\end{tabular}




\begin{tabular}{|c|c|c|c|c|c|}
\hline \multicolumn{6}{|c|}{ Grasas saturadas (\%ET/día) } \\
\hline Modelo 0 & $6,58(6,10 ; 7,06)$ & $6,68(6,15 ; 7,21)$ & $6,68(5,51 ; 7,85)$ & $0,07(-0,46 ; 0,60)$ & 0,800 \\
\hline Modelo 1 & $6,65(6,16 ; 7,14)$ & $6,67(6,13 ; 7,21)$ & $6,61(5,41 ; 7,80)$ & $-0,01(-0,55 ; 0,54)$ & 0,980 \\
\hline Modelo 2 & $6,64(6,07 ; 7,20)$ & $6,33(5,70 ; 6,95)$ & $6,48(5,25 ; 7,70)$ & $-0,16(-0,76 ; 0,44)$ & 0,607 \\
\hline Modelo 3 & $6,63(6,06 ; 7,20)$ & $6,34(5,72 ; 6,97)$ & $6,50(5,27 ; 7,74)$ & $-0,14(-0,75 ; 0,48)$ & 0,659 \\
\hline Modelo 4 & $6,62(6,05 ; 7,19)$ & $6,35(5,73 ; 6,97)$ & $6,55(5,32 ; 7,78)$ & $-0,11(-0,72 ; 0,50)$ & 0,716 \\
\hline \multicolumn{6}{|c|}{ Grasas mono saturadas (\%ET/día) } \\
\hline Modelo 0 & $7,84(7,27 ; 8,41)$ & $8,17(7,54 ; 8,80)$ & $7,74(6,34 ; 9,13)$ & $0,11(-0,52 ; 0,74)$ & 0,732 \\
\hline Modelo 1 & $7,81(7,23 ; 8,39)$ & $8,22(7,59 ; 8,84)$ & $7,63(6,23 ; 9,02)$ & $0,12(-0,52 ; 0,76)$ & 0,710 \\
\hline Modelo 2 & $7,81(7,14 ; 8,47)$ & $7,90(7,17 ; 8,62)$ & $7,57(6,14 ; 9,00)$ & $-0,04(-0,75 ; 0,67)$ & 0,906 \\
\hline Modelo 3 & $7,82(7,15 ; 8,49)$ & $7,87(7,13 ; 8,60)$ & $7,51(6,06 ; 8,96)$ & $-0,09(0,81 ; 0,64)$ & 0,815 \\
\hline Modelo 4 & $7,80(7,14 ; 8,47)$ & $7,90(7,17 ; 8,63)$ & $7,58(6,13 ; 9,02)$ & $-0,04(-0,75 ; 0,68)$ & 0,920 \\
\hline \multicolumn{6}{|c|}{ Grasas polinsaturadas (\%ET/día) } \\
\hline Modelo 0 & $3,91(3,60 ; 4,21)$ & $4,08(3,75 ; 4,42)$ & $4,32(3,57 ; 5,07)$ & $0,20(-0,14 ; 0,53)$ & 0,257 \\
\hline Modelo 1 & $3,77(3,49 ; 4,05)$ & $4,15(3,84 ; 4,45)$ & $4,33(3,65 ; 5,00)$ & $0,32(0,02 ; 0,63)$ & 0,039 \\
\hline Modelo 2 & $3,74(3,41 ; 4,07)$ & $4,07(3,71 ; 4,43)$ & $4,28(3,58 ; 5,00)$ & $0,29(-0,05 ; 0,64)$ & 0,097 \\
\hline Modelo 3 & $3,76(3.43 ; 4,09)$ & $4,08(3,71 ; 4,44)$ & $4,27(3,55 ; 4,98)$ & $0,28(-0,08 ; 0,64)$ & 0,123 \\
\hline Modelo 4 & $3,73(3,40 ; 4,06)$ & $4,07(3,71 ; 4,43)$ & $4,29(3,58 ; 5,00)$ & $0,30(-0,05 ; 0,65)$ & 0,094 \\
\hline \multicolumn{6}{|c|}{ Carbohidratos complejos (\%ET/día) } \\
\hline Modelo 0 & $57,8(56,3 ; 59,3)$ & $56,8(55,2 ; 58,4)$ & $58,4(54,7 ; 62,0)$ & $-0,25(-1,91 ; 1,40)$ & 0,764 \\
\hline Modelo 1 & $57,8(56,3 ; 59,3)$ & $56,7(55,0 ; 58,3)$ & $58,6(55,0 ; 62,2)$ & $-0,22(-1,89 ; 1,44)$ & 0,792 \\
\hline Modelo 2 & $57,8(56,1 ; 59,6)$ & $57,0(55,1 ; 58,9)$ & $58,8(55,0 ; 62,6)$ & $0,04(-1,83 ; 1,90)$ & 0,969 \\
\hline Modelo 3 & $57,9(56,1 ; 59,6)$ & $57,0(55,0 ; 58,9)$ & $58,7(54,9 ; 62,6)$ & $0,01(-1,89 ; 1,92)$ & 0,989 \\
\hline Modelo 4 & $57,9(56,1 ; 59,6)$ & $57,0(55,1 ; 58,9)$ & $58,7(54,9 ; 62,5)$ & $-0,00(-1,89 ; 1,88)$ & 0,997 \\
\hline \multicolumn{6}{|c|}{ Carbohidratos simples (\%ET/día) } \\
\hline Modelo 0 & $18,6(17,2 ; 20,0)$ & $19,5(18,0 ; 21,0)$ & $17,1(13,8 ; 20,5)$ & $0,01(-1,51 ; 1,54)$ & 0,987 \\
\hline Modelo 1 & $18,4(17,1 ; 19,8)$ & $19,4(17,9 ; 20,9)$ & $17,6(14,3 ; 20,9)$ & $0,21(-1,29 ; 1,72)$ & 0,779 \\
\hline Modelo 2 & $18,6(17,0 ; 20,2)$ & $19,2(17,4 ; 20,9)$ & $17,6(14,1 ; 21,0)$ & $-0,11(-1,81 ; 1,60)$ & 0,901 \\
\hline Modelo 3 & $18,6(17,0 ; 20,3)$ & $19,2(17,4 ; 20,9)$ & $17,6(14,1 ; 21,1)$ & $-0,13(-1,88 ; 1,62)$ & 0,884 \\
\hline Modelo 4 & $18,7(17,1 ; 20,3)$ & $19,2(17,4 ; 20,9)$ & $17,4(13,9 ; 20,8)$ & $-0,25(-1,98 ; 1,48)$ & 0,776 \\
\hline \multicolumn{6}{|c|}{ Alcohol (\%ET/día) } \\
\hline Modelo 0 & $33,1(29,0 ; 37,3)$ & $36,4(31,8 ; 41,0)$ & $35,8(25,5 ; 46,1)$ & $2,17(-2,40 ; 6,73)$ & 0,351 \\
\hline Modelo 1 & $34,1(30,0 ; 38,3)$ & $39,5(34,9 ; 44,0)$ & $37,6(27,6 ; 47,6)$ & $3,23(-1,30 ; 7,76)$ & 0,162 \\
\hline Modelo 2 & $34,6(29,9 ; 39,3)$ & $40,9(35,8 ; 46,1)$ & $37,6(27,5 ; 47,7)$ & $3,10(-1,85 ; 8,05)$ & 0,219 \\
\hline Modelo 3 & $34,4(29,7 ; 39,2)$ & $40,3(35,2 ; 45,5)$ & $37,1(27,0 ; 47,3)$ & $2,89(-2,15 ; 7,93)$ & 0,260 \\
\hline Modelo 4 & $34,6(29,9 ; 39,3)$ & $41,1(35,9 ; 46,3)$ & $38,0(27,7 ; 48,2)$ & $3,36(-1,67 ; 8,39)$ & 0,189 \\
\hline
\end{tabular}

Datos presentados como promedio y $95 \%$ IC según genotipo. El modelo genético aditivo indica el promedio de incremento en la variable de adiposidad por cada copia adicional de la variante de riesgo (A). Este efecto aditivo y su respectivo $95 \%$ IC fue determinado mediante regresión lineal. Los análisis fueron ajustados por: Modelo 0 - sin ajustar; Modelo 1 -ajustado por edad, sexo, etnia, nivel educacional, ingreso económico, nivel socioeconómico, y zona de residencia (urbano/rural); Modelo 2 - ajustado por el modelo 1 pero también por actividad física, tiempo sedente y tabaquismo; Modelo 3 - fue ajustado por el Modelo 2 pero también por IMC; y Modelo 4 - ajustado por Modelo 2 y perímetro de cintura. 


\section{Discusión}

Numerosos trabajos han explorado si el grFTO se asocia con una ingesta aumentada de nutrientes y energía, observándose resultados inconsistentes entre ellos e incluyendo un amplio espectro de asociaciones con: aumento de la ingesta energética total, de grasas saturadas, de proteínas, de carbohidratos o ausencia de relación con ingesta de macronutrientes e ingesta energética ${ }^{28}$. Los datos obtenidos en este trabajo corroboran que los portadores del polimorfismo estudiado, presentan una mayor ingesta energética.

En un intento por articular la evidencia publicada, dos revisiones sistemáticas determinaron que el grFTO está asociado a un mayor porcentaje de energía obtenida a partir de proteínas y un menor consumo de energía total ${ }^{18,29}$. Este último hallazgo resulta inesperado considerando que el grFTO ha sido consistentemente asociado a un mayor IMC y a parámetros de adiposidad alterados en la mayoría de las poblaciones estudiadas ${ }^{7,9}$. Una de las limitaciones que mencionan estos estudios es que no fue posible identificar cuáles fueron los alimentos que contribuían a este mayor consumo de proteínas (Ej., consumo de carnes procesadas, rojas, $\mathrm{u}$ otros alimentos con alto contenido de proteínas). Al respecto, en ambas revisiones sistemáticas se manifiestan aprensiones respecto a la precisión de los métodos de registro de los alimentos que consisten mayoritariamente en cuestionarios de frecuencia alimentaria (FFQ) o bitácoras de alimentación ${ }^{18,29}$, las cuales son frecuentemente cuestionadas por su alto grado de inexactitud derivada del menor reporte energético declarado especialmente por obesos ${ }^{30}$. En este contexto, se podría suponer que la falta de asociación entre el grFTO e ingesta energética elevada identificada en algunos estudios ${ }^{18,29}$, podría estar asociada al reporte incompleto de los FFQs.

Estudios previos, realizados principalmente en poblaciones europeas, han demostrado una asociación de grFTO con alimentación, existiendo muy pocos trabajos realizados en población latina, entre los que se encuentran estudios en españoles $^{31,32}$ y brasileños ${ }^{33}$.

Debido a la complejidad de la relación entre genotipo y alimentación, este tipo de asociaciones puede ser modulado por múltiples variables. Por ejemplo, es conocido que el repertorio genético particular de un individuo puede modular la interrelación de FTO con el hambre y la saciedad postprandial, como es el caso de los polimorfismos rs992472 en DNMT3B y rs1137101 en $L E P R^{34}$. Por esta razón es importante verificar si la asociación previamente observada en poblaciones europeas es válida en población chilena, la cual posee su propio repertorio genético y está sometida a condiciones ambientales particulares, como disponibilidad de alimentos y costumbres culinarias. En este sentido, los resultados presentados en este trabajo muestran una posible asociación entre el gen FTO con un mayor consumo energético en población chilena. Considerando que individuos portadores del alelo de riesgo podrían consumir entre 136 a $173 \mathrm{kcal} /$ día más que aquellos sin el alelo de riesgo, los individuos portadores presentarían una mayor predisposición a desarrollar obesidad ya que, si bien el efecto aislado del consumo de 136 a $173 \mathrm{kcal} /$ día es pequeño, el efecto aditivo de este consumo en un mes (hasta $5.190 \mathrm{kcal}$ extras) o en un año (hasta $63.145 \mathrm{kcal}$ extras) es el que podría presentar una repercusión directa en los niveles de adiposidad y, por ende, aumentar la probabilidad de desarrollar obesidad. Esta hipótesis está respaldada por estudios de intervención donde se ha otorgado una dieta hipercalórica equivalente a $66.156 \mathrm{kcal}$ en el transcurso de 8 semanas, la cual provocó un aumento de $7,6 \mathrm{~kg}$ en peso corporal en los participantes ${ }^{35}$.

Entre las fortalezas de este trabajo se encuentra la utilización del registro por pesada de alimentos para determinar la ingesta energética en lugar de los FFQs, los cuales presentan un mayor sesgo por subestimación de ingesta, lo cual podría explicar la falta de asociación identificada en estudios previos que han utilizado esta metodología. Otra fortaleza del estudio es el ajuste por una amplia cantidad de variables de confusión, lo cual permite establecer si las asociaciones observadas son independientes de estos factores. Entre las limitaciones, es importante destacar que el reducido tamaño de la muestra pudo haber limitado el poder estadístico y ocultado algunas asociaciones con otros nutrientes. No obstante, el tamaño muestral fue suficiente para establecer una relación estadísticamente significativa e independiente de factores de confusión entre el consumo energético elevado y la presencia del grFTO. Además, el limitado número de personas del estudio impidió realizar análisis comparativos entre los portadores de ascendencia mapuche y europea. 


\section{Conclusión}

El presente estudio muestra por primera vez una asociación positiva entre el grFTO y mayor consumo energético en población adulta chilena, independiente de las variables de confusión. A pesar del posible rol de esta asociación en el desarrollo de obesidad, es importante considerar la naturaleza multifactorial de esta patología, en la cual los estilos de vida tienen un rol fundamental en su curso y desarrollo. Por lo tanto, si bien individuos con el alelo de riesgo podrían presentan una mayor ingesta energética, mantener estilos de vida saludables como la práctica de actividad física regular y/o una alimentación equilibrada podrán reducir la predisposición genética a la obesidad.

\section{Referencias}

1. Collaboration NCDRF. Trends in adult body-mass index in 200 countries from 1975 to 2014: a pooled analysis of 1698 population-based measurement studies with 19.2 million participants. Lancet. 2016; 387 (10026): 1377-96.

2. WHO. 10 datos sobre la obesidad. World Health Organization. 2017; Disponible: http://www.who.int/ features/factfiles/obesity/es/.

3. Petemann F, Durán E, Labraña AM, Martínez MA, Leiva AM, Garrido-Méndez A, et al. Factores asociados al desarrollo de obesidad en Chile: resultados de la Encuesta Nacional de Salud 2009-2010. Rev Med Chile 2017; 145 (6).

4. López-Sobaler AM, Rodríguez-Rodríguez E, Aranceta-Bartrina J, Gil A, González-Gross M, Serra-Majem L, et al. General and Abdominal Obesity Is Related to Physical Activity, Smoking and Sleeping Behaviours and Mediated by the Educational Level: Findings from the ANIBES Study in Spain. PloS one 2016; 11 (12): e0169027.

5. Allison DB, Kaprio J, Korkeila M, Koskenvuo M, Neale MC, Hayakawa K. The heritability of body mass index among an international sample of monozygotic twins reared apart. International journal of obesity and related metabolic disorders: journal of the International Association for the Study of Obesity 1996; 20 (6): 501-6.

6. Loos RJ. The genetics of adiposity. Curr Opin Genet Dev 2018; 50: 86-95.

7. Frayling TM, Timpson NJ, Weedon MN, Zeggini E, Freathy RM, Lindgren CM, et al. A common variant in the FTO gene is associated with body mass index and predisposes to childhood and adult obesity. Science 2007; 316 (5826): 889-94.

8. Petermann F, Villagrán M, Troncoso C, Mardones L, Leiva AM, Martínez MA, et al. Association between polymorphism rs9939609 in FTO gene and adiposity markers in the chilean adult population. Rev Med Chile 2018.

9. Riffo B, Asenjo S, Saez K, Aguayo C, Muñoz I, Bustos $\mathrm{P}$, et al. FTO gene is related to obesity in Chilean Amerindian children and impairs HOMA-IR in prepubertal girls. Pediatr Diabetes 2012; 13 (5): 384-91.

10. Wardle J, Llewellyn C, Sanderson S, Plomin R. The FTO gene and measured food intake in children. International journal of obesity (2005) 2009; 33 (1): 42-5.

11. Wardle J, Carnell S, Haworth CM, Farooqi IS, O'Rahilly $\mathrm{S}$, Plomin R. Obesity associated genetic variation in FTO is associated with diminished satiety. The Journal of clinical endocrinology and metabolism 2008; 93 (9): 3640-3.

12. Timpson NJ, Emmett PM, Frayling TM, Rogers I, Hattersley AT, McCarthy MI, et al. The fat mass- and obesity-associated locus and dietary intake in children. The American journal of clinical nutrition 2008; 88 (4): 971-8.

13. Tanofsky-Kraff M, Han JC, Anandalingam K, Shomaker LB, Columbo KM, Wolkoff LE, et al. The FTO gene rs9939609 obesity-risk allele and loss of control over eating. The American journal of clinical nutrition. 2009; 90 (6): 1483-8.

14. Cecil JE, Tavendale R, Watt P, Hetherington MM, Palmer CN. An obesity-associated FTO gene variant and increased energy intake in children. N Engl J Med 2008; 359 (24): 2558-66.

15. Qi Q, Downer MK, Kilpelainen TO, Taal HR, Barton SJ, Ntalla I, et al. Dietary Intake, FTO Genetic Variants, and Adiposity: A Combined Analysis of Over 16,000 Children and Adolescents. Diabetes 2015; 64 (7): 2467 76.

16. Tung YC, Ayuso E, Shan X, Bosch F, O'Rahilly S, Coll AP, et al. Hypothalamic-specific manipulation of Fto, the ortholog of the human obesity gene FTO, affects food intake in rats. PLoS One 2010; 5 (1): e8771.

17. Tanaka T, Ngwa JS, van Rooij FJ, Zillikens MC, Wojczynski MK, Frazier-Wood AC, et al. Genome-wide meta-analysis of observational studies shows common genetic variants associated with macronutrient intake. The American journal of clinical nutrition 2013; 97 (6): 1395-402.

18. Livingstone KM, Celis-Morales C, Lara J, Ashor AW, Lovegrove JA, Martínez JA, et al. Associations between FTO genotype and total energy and macronutrient 
intake in adults: a systematic review and meta-analysis. Obes Rev 2015; 16 (8): 666-78.

19. Steemburgo T, Azevedo MJ, Gross JL, Milagro FI, Campion J, Martínez JA. The rs9939609 polymorphism in the FTO gene is associated with fat and fiber intakes in patients with type 2 diabetes. J Nutrigenet Nutrigenomics 2013; 6 (2): 97-106.

20. Speakman JR, Rance KA, Johnstone AM. Polymorphisms of the FTO gene are associated with variation in energy intake, but not energy expenditure. Obesity (Silver Spring) 2008; 16 (8): 1961-5.

21. OECD. Obesity Update 2017. Organization for Economic Co-operation and Development. 2017.

22. Atalah SE. Epidemiología de la obesidad en chile. Revista Médica Clínica Las Condes 2012; 23 (2): 117-23.

23. Celis-Morales CA, Perez-Bravo F, Ibanes L, Sanzana R, Hormazabal E, Ulloa N, et al. Insulin resistance in Chileans of European and indigenous descent: evidence for an ethnicity $\mathrm{x}$ environment interaction. PloS one 2011; 6 (9): e24690.

24. WHO. Obesity: preventing and managing the global epidemic. World Health Organization. 2000;Disponible en: http://www.who.int/nutrition/publications/obesity/ WHO_TRS_894/en/.

25. Durnin JV, Womersley J. Body fat assessed from total body density and its estimation from skinfold thickness: measurements on 481 men and women aged from 16 to 72 years. British journal of nutrition 1974; 32 (1): 77-97.

26. Buckley J, Sim J, Eston R, Hession R, Fox R. Reliability and validity of measures taken during the Chester step test to predict aerobic power and to prescribe aerobic exercise. British Journal of Sports Medicine 2004; 38 (2): 197-205.

27. Freedson PS, Melanson E, Sirard J. Calibration of the Computer Science and Applications, Inc. accelerometer.
Medicine and science in sports and exercise 1998; 30 (5): 777-81.

28. Speakman JR. The 'Fat Mass and Obesity Related' (FTO) gene: Mechanisms of Impact on Obesity and Energy Balance. Curr Obes Rep 2015; 4 (1): 73-91.

29. Qi Q, Kilpelainen TO, Downer MK, Tanaka T, Smith $\mathrm{CE}$, Sluijs I, et al. FTO genetic variants, dietary intake and body mass index: insights from 177,330 individuals. Hum Mol Genet 2014; 23 (25): 6961-72.

30. Dhurandhar NV, Schoeller D, Brown AW, Heymsfield SB, Thomas D, Sorensen TI, et al. Energy balance measurement: when something is not better than nothing. Int J Obes (Lond) 2015; 39 (7): 1109-13.

31. Corella D, Arnett DK, Tucker KL, Kabagambe EK, Tsai $\mathrm{M}$, Parnell LD, et al. A high intake of saturated fatty acids strengthens the association between the fat mass and obesity-associated gene and BMI. J Nutr 2011; 141 (12): 2219-25.

32. Galbete C, Toledo J, Martínez-González MA, Martínez JA, Guillen-Grima F, Marti A. Lifestyle factors modify obesity risk linked to PPARG2 and FTO variants in an elderly population: a cross-sectional analysis in the SUN Project. Genes Nutr 2013; 8 (1): 61-7.

33. Lourenco BH, Qi L, Willett WC, Cardoso MA, Team AS. FTO genotype, vitamin D status, and weight gain during childhood. Diabetes 2014; 63 (2): 808-14.

34. den Hoed M, Westerterp-Plantenga MS, Bouwman FG, Mariman EC, Westerterp KR. Postprandial responses in hunger and satiety are associated with the rs9939609 single nucleotide polymorphism in FTO. Am J Clin Nutr 2009; 90 (5): 1426-32.

35. Johannsen DL, Tchoukalova Y, Tam CS, Covington JD, Xie W, Schwarz JM, et al. Effect of 8 weeks of overfeeding on ectopic fat deposition and insulin sensitivity: testing the "adipose tissue expandability" hypothesis. Diabetes Care 2014; 37 (10): 789-97. 